\title{
39
}

\section{Dynamic rate adaption for efficient use of frame relay network}

\author{
A. Calveras Augé, J. Paradells \\ Dept. Mat. Apl. i Telemàtic. Universitat Politècnica de \\ Catalunya \\ Mod. C3 C/ Jordi Girona 1-3, 08034 Barcelona, SPAIN \\ Tel: +34 3 401.60.13, Fax: +34 3 401.59.81, \\ e-mail:\{acalveras, teljpa\}@mat.upc.es \\ Jordi Puga Antequera \\ Sema Group sae \\ Provença, 388,08025 Barcelona, SPAIN \\ Klaus-Jürgen Schulz \\ European Space Agency (ESA) \\ European Space Operations Centre (ESOC) \\ Robert-Bosch-Str. 5, D-64293 Darmstadt, GERMANY
}

\begin{abstract}
Modern network services like Frame Relay provide information about the load or congestion state of the network in real time to all attached end-systems. This allows the end-systems to inject traffic into the network above the agreed service contract based on committed information rate and burst excess size. This paper describes a rate control algorithm that is added to the traditional TCP transport protocol
\end{abstract}

Broadband Communications P. Kühn \& R. Ulrich (Eds.)

(C) 1998 IFIP. Published by Chapman \& Hall 
elements which exploits the congestion state information conveyed in the Forward/Backward Explicit Congestion Notification (FECN/BECN) of frame relay. The simulated network and transport protocol behaviour for typical applications of the European Space Agency ${ }^{*}$ shows a win-win situation for the network user and the network provider.

\section{Keywords \\ Frame Relay, TCP, Rate Control, Congestion Avoidance}

\section{INTRODUCTION}

The European Space Agency (ESA) in co-operation with Sema Group sae and Polytechnic University of Catalonia has investigated the use of frame relay network services for distribution of high rate telemetry between satellite receiving ground stations or other space data entry points and remote processing sites. The objective of this study is to connect telemetry end systems directly to the frame relay network in order to make efficient use of the frame relay inherent features of congestion avoidance, which would not have been possible by just connecting Internet Protocol routers to the frame relay network. The system shall allow a data rate of $2 \mathrm{Mbps}$ and over per subscriber.

The study started from a detailed definition of the application service semantic specific to telemetry distribution applications, then identified a complete OSI like protocol stack above frame relay, thereby emphasising on the study of protocol elements in the transport layer for a high speed network scenario. In order to exploit the frame relay congestion avoidance mechanism a new protocol element for rate control was specified for the transport layer.

The complete system, i.e. 2 end-systems, a network with 3 nodes and additional background traffic was investigated in various simulation scenarios.

\section{SERVICE DEFINITION}

This study, based on the service definitions (volume 1 and 2, 1992), involves the transfer of information between different space agencies, in order to ensure uniformity in the Service Interface.

Telemetry Application Services require that data are relayed complete, sequence preserved, and without errors. Therefore, a protocol stack with reliable transport services.

\footnotetext{
* Part of this work is the result of a study carried out for the European Space Agency under contract: 10687/94/D/DK(SC)
} 


\section{FRAME RELAY USER NETWORK INTERFACE}

Frame Relay (FR) according to Smith (1992) and Black (1992) is a network access protocol based on a fast packet switching technology, connection oriented and that preserves the transmission frame sequence. Two characteristics draw its advantages: low overhead due to minimal node processing, and the ability to support traffic bursts. Error control for incorrectly received frames and flow control processing are largely absent.

In present networks, losses are mainly due to congestion. FR has a congestion notification mechanism but a congestion control is not foreseen. So, the key point in it is the policy applied to avoid congestion, and the mechanism to notify the users when it occurs.

FR offers a range of options at subscription time: Access Rate (AR), Committed Information Rate (CIR) that provides his foreseen minimum guarantied data throughput, and Excess Information Rate (EIR) that represents the potential peak data flows.

Once congestion occurs, FR sends a notification and provides the possibility of selecting frames to be discarded (by means of FECN, BECN and DE bits respectively).

\section{SIMULATION ENVIRONMENT}

This section describes the models used in the simulation based study.

\subsection{Application Service Modelling}

CCSDS (Consultative Committee on Space Data Systems) Packets arrive at the Service Provider Application from the Space Link Services, and they are stored in the Ground Station in an infinite buffer until they are transmitted.

A general packet generation with a Poisson distribution has been assumed, with two functional modes having different inter-arrival packet mean: Burst mode and Non burst mode. The packet length is assumed to has a uniform distribution as the one given by Johnson (1991)

\subsection{User Network Interface (UNI)}

The User Network Interface (UNI) is the access point of the Protocol Stack to the network. It is modelled as an entity that accepts packets from higher level layers, and encapsulates them in FR frames that are injected in the network.

The UNI controls the maximum burst rate that the FR network can accept by means of the FR parameters: CIR, EIR and AR. 


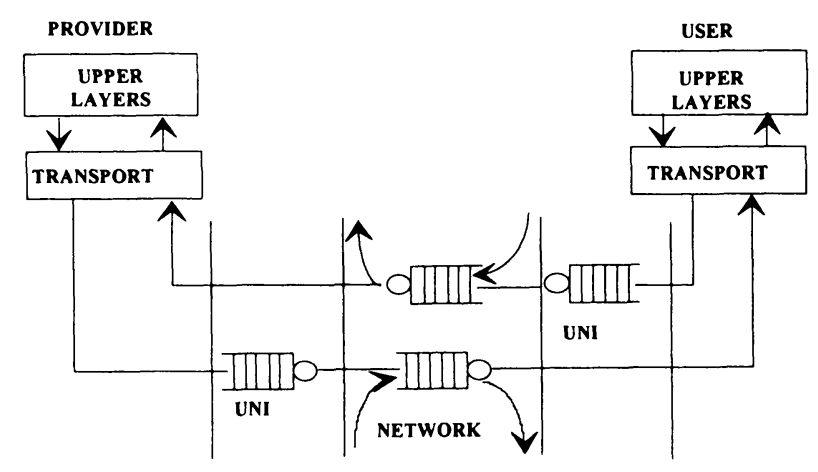

Figure 1 Layered Architecture, UNI and FR Network.

\subsection{Network Modelling}

FR defines an access interface to the network but not the way this network is implemented. The modelled network provides FR congestion notification (BECN and FECN bits) and a discarding strategy based in the DE bit.

A model with three nodes has been chosen for the simulation. Each node is modelled by means of a server with a finite buffer. The service time represents the packet processing time plus the transmission time, and the buffer length threshold is used to model the congestion effect.

Background traffic with exponential distribution is considered, and it also reacts to congestion. The user traffic flowing through a virtual circuit represents the $33 \%$ of the total traffic.

The parameters that define the network model are:

- Trunk Throughput: 3.285 Mbps.

- Propagation delay: Local $(5 \mathrm{~ms})$, intercontinental $(50 \mathrm{~ms})$ and satellite link (250 ms).

- Buffers capacity: it defines the probability of network congestion. These buffers have been designed in the model in such way that the congestion effect appears frequently to study its consequences.

\section{Congestion modelling}

Congestion occurs when buffers are full. However, congestion notification is sent when buffers reach $80 \%$ of its capacity.

To model the effect of congestion when a buffer reaches the $100 \%$ of its capacity, a random number of frames in the buffer with the DE bit set are discarded, and the rest of the frames are marked with the DE bit, and consequently will be discarded if necessary in next congestion situations. 


\section{TRANSPORT LAYER}

This section presents the studied Protocol Profiles and Elements, for the particular case of telemetry applications and assuming to be used on top of a FR Network. The eXpress Transfer Protocol (XTP) and the Transmission Control Protocol (TCP) were selected as the main candidates, and both were simulated. Although XTP is more modern no congestion avoidance mechanisms have been developed for it. Meanwhile, TCP starts from a classical protocol, but it has been studied in detail, and extensions for congestion control and high speed environments have been added.

In consequence, TCP protocol elements have been adopted as a baseline for the final solution and then adapted to FR networks. Several options within the current analysis work, including extensions and modifications to adapt it to FR networks have been considered.

\subsection{Extended TCP Over Frame Relay}

This section mainly describes the extended TCP Elements and the additional BECN congestion control algorithms that are most suitable in the Telemetry Delivery Applications Services over FR. The usage of BECN in the congestion control mechanism is something new, not yet totally studied.

TCP as it is decribed by Postel (1981) is a connection oriented protocol that was designed to provide end-to-end reliable data delivery. Although it uses Positive Acknowledgement with Retransmission, a Selective Repeat scheme has also been modelled as a possible extension of the protocol (Fall, 1995) (Mathis, 1996).

Accurate dynamic determination of the retransmission time-out (RTO) interval seems to be essential to TCP performance (Jacobson, 1988). RTO is determined by estimating the mean and variance of the measurement round-trip time (RTT), i.e., the time interval between sending a segment and receiving an acknowledgement for it. To avoid the ambiguity in the estimate RTO, the Karn's Algorithm (Jacobson, 1988) is also implemented, combined with an exponential backoff strategy for the retransmission timer. Many TCP implementations base their RTT measurements upon a sample of only one packet per window, which results in an unacceptable poor RTT estimate when the bandwidth-delay product is large. An extension to TCP can solve these problems. Using the TCP header options, the sender places a TimeStamp (Jacobson, 1992) in each data segment, which is sent back in acknowledgements.

A credit-based adaptive window-sliding mechanism is used for end-to-end flow control, and the algorithms known as Slow-Start and Congestion Avoidance (Jacobson, 1988), are used for congestion control. They operate by observing that the rate at which new packets should be injected into the network is the rate at which the acknowledgements are returned by the other end. Upon detecting congestion, the sender drastically reduces the window size and slowly starts to 
increase the rate at which it transmits packets, back up to its previous levels. This approach reduces the probability of packet retransmission, and increases the effective throughput of the connection.

The BECN Congestion Algorithm (Jubainville, 1994) has been added as an extension, to take full advantage of FR congestion indication bits. Usually, these bits are not used by the upper layers although they are present in the protocol.

This algorithm is based in the fact that FR sets the FECN and BECN bits to indicate that congestion is occurring or has occurred in the network. The user detects these indications and assumes that the network is congested and reduces or suspends the transmission until the network recovers from the congested situation.

When a node becomes congested, FR sets these bits in each direction of the connection, in all the frames that are in transit in the node. If there is no reversing data flowing in the reverse direction of data flow, the FR Consolidated Link Layer Management (CLLM) message is used as a congestion notification message that can be forwarded to the UNI as required.

The user response to the BECN or CLLM congestion indication has been studied at the Transport Layer, introducing a Rate Control Mechanism at this level.

For the BECN algorithm a step count (S), is used to determine when the protocol should take congestion avoidance action.

When the UNI receives a frame with the BECN (or CLLM) bit set, data sent to the transport layer contains an indication of congestion. The procedure to be adopted by the sender on receipt of this indication is as follows:

1. If TCP's current offered data rate is greater than the agreed CIR, TCP reduces his offered rate to the CIR agreed for the circuit.

2. If a number of consecutive indications equal to the step count are received, TCP should reduce his offered rate to the next "step" rate below the current rate. This continues with a rate reduction to the next "step" rate after every $S$ consecutive indications.

The "step" proposed rates are:

- $0.675 * C I R$

- $0.5 * C I R$

- $0.25 * C I R$

3. After having reduced the offered rate, the user may increase this rate by 0.125 times the throughput, after any $\mathrm{S} / 2$ consecutive received data without congestion indications.

Other studies based on TCP and Explicit Congestion Notification are of interest. Floyd (1994) proposes the guidelines for TCP's response to Explicit Congestion Notification Mechanisms, Calhoun (1995) discusses Congestion Management in the IPV6 Internetworks, and Schulzrine (1996) proposes an enhancement to the TCP's congestion control mechanisms using binary congestion notification.

Another extension is added to the TCP, the Fast Recovery and Fast Retransmit algorithm (Stevens, 1997). In the TCP error recovery mechanism, a received out of sequence segment generates immediately a duplicated ACK. Since FR does not 
disorder packets, a duplicated ACK means that data is lost. Nevertheless, it has to be pointed out that the RTO expiration of a packet that has only been delayed but not lost, may cause a received out of sequence segment and consequently a duplicated ACK to be received. In this case the reception of a duplicated ACK does not mean that a packet has been lost.

Nevertheless, if a number of duplicated ACK's are received in a row, it is a strong indication that a segment has been lost. A retransmission of what appears to be the missing segment is performed then, without waiting for the RTO to expire. In this case, the TCP congestion avoidance algorithm is also performed, but not the slow start. In the specific case of the study the number of duplicates that force retransmission is two.

\section{DISCUSSION}

The selection of the protocol stack has been validated by means of computer based simulation techniques. Results and conclusions are valid for the particular case of Telemetry Applications on top of FR, and a particular decision about each Protocol Element is provided.

\subsection{Environment}

The obtained results for the evaluation are obtained using the following parameters:

- Source Parameters:

- Burst Packet inter-arrival: 4,87 msec

- $\quad$ Non Burst Packet inter-arrival:19,46msec

- Burst Rate Duration: 1 sec

- Non Burst rate Duration: $2 \mathrm{sec}$

- Packet Length:

- Maximum: 2000 octets, Minimum: 500 octets, Average: 1250 octets

- Transport Layer:

- MSS: 1560 octets

- Network:

- Transmission time: 3,9 msec, Node Buffer Length: 50 frames

- UNI:

- $\quad A R: 2 M b p s, C I R: 1 M b p s$

The performance measurements are:

- Application efficiency (\%):

It is the ratio of amount of data generated by the application to that injected in the network.

- Frame Losses (\%):

It is the ratio of bits transmitted through the network to those injected in it.

- $\quad$ End to end rate (bps) 


\subsection{Fast Retransmit And Fast Recovery}

TCP extensions for high performance protocols, propose another error control and recovery, to act quickly when losses occur at the network.

One advantage of this algorithm is to transform the Go-Back-N retransmission scheme to a "quasi-selective" retransmission mechanism, avoiding a large number of unnecessary retransmitted packets.

Consequently, it is recommended to use the fast retransmission and fast recovery option, mainly, when bandwidth-delay product is high. Nevertheless, when a fixed retransmission timer is considered as the best solution to avoid unnecessary retransmissions (this concept will be discussed in following sections), the Fast Retransmission and Fast Recovery Algorithm are then mandatory.

Table 1 shows these ideas. The parameters and protocol profiles are the same as before but the BECN Algorithm that is not implemented.

Table 1 Fast Retransmit and Fast Recovery effect on application efficiency, frame losses and end-to-end rate.

Mechanism used Application efficiency Frame losses (\%) End-to-end rate (Mbps)

\begin{tabular}{llll}
\hline $\begin{array}{l}\text { Without Fast ret. } \\
\text { and fast reco. }\end{array}$ & 89,23 & 1,7 & 0,973 \\
\hline $\begin{array}{l}\text { With Fast ret. } \\
\text { and fast reco. }\end{array}$ & 91,97 & 0,65 & 0,962 \\
\hline
\end{tabular}

\subsection{Congestion Control}

Congestion control mechanisms in the Transport Layer are mandatory, since FR only notifies and discards packets in case of congestion, and this is the main cause of data losses in networks with very low bit error rates.

It has been shown how each type of congestion control affects the protocol performance: TCP incorporated Congestion Control Mechanisms and BECN Congestion Control Mechanism. Table 2 shows the results.

Both congestion control mechanisms are recommended since:

- BECN congestion algorithm prevents the network from congestion situations to occur, without reducing drastically the offered traffic.

- When frames are lost due to congestion, the TCP congestion control reduces drastically the offered rate. 
Table 2 Congestion Control Mechanisms effect on the performance parameters used

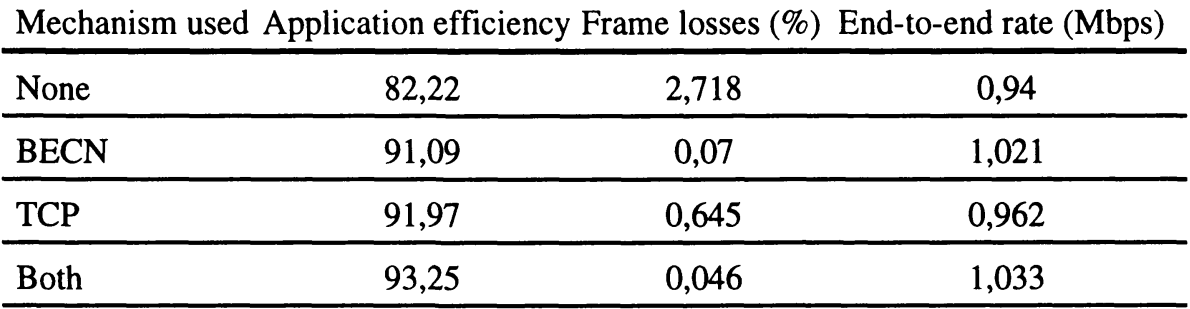

It can be seen that the combination of both mechanisms improves the results. The usage of BECN alone offers a significant performance.

\subsection{RTO And RTT Measurement}

The RTT Measurement algorithm updates the Retransmission timer, adjusting it dynamically to the state of the network. This measure is adequate when the bandwidth-delay product is low, and the measure really shows the actual state of the network, but in case a high bandwidth-delay product, a more accurate measure has to be made to avoid unnecessary retransmissions. The TimeStamp facility may be optionally used to increase the accuracy of that measure.

Another important point is that unnecessary retransmissions have been shown to affect the fast retransmission and fast recovery algorithm, as well the TCP congestion control algorithm. Therefore, it is important to avoid these unnecessary retransmissions.

After the simulation studies taking into account the bursty traffic scheme and the network topology, the conclusions about the RTT measurements and the RTO dynamic adaptation are:

- As the network delay increases, the dynamic measurement of the retransmission timer is not adequate, since the actual network congestion situation in which the new timer acts, is rather different from the situation in which the timer was measured.

- Although the TimeStamp option gives a more accurate RTT, this optional mechanism is not sufficient to avoid unnecessary retransmissions.

In conclusion, the use of the RTT measurement algorithm is not recommended, specially when bandwidth-delay product is significant. Therefore, a solution in this case is to consider a fixed RTO that should be greater than the maximum expected RTT, being long enough to avoid the unnecessary retransmissions.

Nevertheless, the use of a fixed RTO has to be complemented with the use of the Fast Retransmission and Fast Recovery Algorithm. In this case, the algorithm forces the retransmission of the lost packet before the timer expiration.

The results are shown in the following tables for fixed and dynamic RTO. 
Table 3 The effect on the performance of the RTT measurement and the RTO adjustment.

Mechanism used Application efficiency Frame losses (\%) End-to-end rate (Mbps)

\begin{tabular}{llll}
\hline $\begin{array}{l}\text { Dynamic adjust } \\
\text { of RTO }\end{array}$ & 91,03 & 0,027 & 0,124 \\
\hline $\begin{array}{l}\text { No adjust of } \\
\text { RTO. }\end{array}$ & 95,30 & 0,206 & 0,776 \\
\hline
\end{tabular}

The topology and the type of network (based of frame relay) lead to this recommendation about the usage of a fixed RTO. In a more complex network with the possibility to change routes it is not clear if the solution with fixed RTO could be implemented.

\section{CONCLUSIONS}

The main conclusion of the study is that using the identified Protocol Elements of the extended TCP, and the BECN congestion control algorithm at the Transport Layer is the best option for the delivery of telemetry over FR networks.

The simulation results have allowed to select the most suitable TCP protocol elements and parameters that show the best efficiency, specially in congested networks.

Following graphs show the comparison between two protocol profiles:

Case 1. This protocol profile includes the TCP extensions, the Fast Recovery and Fast Retransmission algorithm, the Go-Back-N error recovery algorithm, considers the BECN Algorithm against congestion, and a fixed RTO. This is the recommended protocol profile.

Case 2. This protocol profile neither considers the TCP extensions for high performance nor the BECN algorithm against congestion situations, and a dynamic RTO.

The percentages of improvement ${ }^{*}$ with the recommended protocol profile are shown in tables 4 and 5.

\footnotetext{
* Percentage of Improvement (\%): percentage the recommended case adds to the other, referred to this case. In the Frame Losses a negative value means an improvement (with a maximum value of $100 \%$ ) and a positive one a worsening.
} 
Table 4 Improvement obtained using the proposed profile

\begin{tabular}{ccc}
\hline Application Efficiency & Frame Losses & End-to-End Rate \\
\hline $17,637 \%$ & $-98,823 \%$ & $8,955 \%$ \\
\hline
\end{tabular}

Table 5 Performance of the protocol with the two cases C1 (case 1) and C2 (case 2) commented above.

Mechanism used Application efficiency Frame losses (\%) End-to-end rate (Mbps)

\begin{tabular}{llll}
\hline Case 1 & 96,72 & 0,03 & 1,0237 \\
\hline Case 2 & 82,22 & 2,72 & 0,9396 \\
\hline
\end{tabular}

\section{FUTURE WORK}

The ATM Forum has specified 5 ATM service classes (ATM Forum, 1996), one of them being the so called Available Bitrate (ABR) service, which includes an explicit rate control mechanism in concept similar to the frame relay FECN/BECN information to convey information about the network load and congestion state from the network to the user. Future work will have to address how the ATM ABR service will interface to the transport layer which would then take care of proper rate adaptation.

The results of the study will now be verified in a real network scenario. ESA has let a contract to Sema Group (Number: 11772/96/D/DK(SC)) for development of the end systems, which will be connected to a frame relay test network.

\section{REFERENCES}

(1992) Description of CCSDS Ground Infrastructure Cross-Support Services. Volume 1: Service Concept (DRAFT). 20th April 1992 (CCSDS-P3V1).

(1992) Description of CCSDS Ground Infrastructure Cross-Support Services. Volume 2: Space Data Services. 4th April 1992 (CCSDS-P3V2).

ATM Forum (1996) Traffic Management Specification, Version 4.0, April 1996.

Black, U. D. (1992) Frame Relay Networks: specifications and implementations. McGraw-Hill.

Calhoun, (1995) Congestion control in IPv6 Internetworks, internet draft, May 1995.

Fall, K.and Floyd, S. (1995) Comparisons of Tahoe, Reno, and Sack TCP. A Postscript version of this document is available from ftp://ftp.ee.lbl.gov/papers/sacks.ps.z, December 1995. 
Floyd, S. (1994) TCP and explicit congestion notification. A Postscript version of this document is available from $\mathrm{ftp}: / / \mathrm{ftp}$.ee.lbl.gov/papers/tcp_ ecn. 4.ps.z, 1994.

Jacobson, V (1992) TCP Extensions for High Performance. RFC 1323. May 1992.

Jacobson, V. (1988). Congestion Avoidance and Control. Computer Communication Review, vol. 18, no. 4, 314-329.

Johnson, M. J. (1991) Coping with data from Space Station Freedom. Computer Networks and ISDN Systems, 22, 131-142.

Jubainville, R. (1994) Congestion control for frame relay. Telecommunications, March 1994, 77-80.

Mathis, M., Mahdavi, J., Floyd, S. and Romanov, A. (1996) TCP Selective Acknowledgements Options. Internet Draft. 1996.

Postel, J. (1981) Transmission control protocol, RFC 793, January 1981.

Schulzrinne, S. H. (1996) Binary Congestion Notification in TCP, IEEE June 25 1996,772-776.

Smith, P. (1992) Frame Relay. Principles and Applications. Addison-Wesley.

Stevens, W (1997) TCP slow start, congestion avoidance, fast retransmit and fast recovery, RFC 2001, Janury 1997.

\section{BIOGRAPHIES}

Anna Calveras obtained the electrical engineering degree from the Polytechnic University of Catalonia. She worked at Sema Group Spain where she participated in several research projects. Later she returned to the University where she holds a position as an assistant professor and currently she is working in her Ph.D. in the optimisation of the TCP protocol on heterogeneous network environments.

Josep Paradells obtained the Ph.D. degree from the Polytechnic University of Catalonia. $\mathrm{He}$ is associate professor at the mentioned University.

Jordi Puga obtained the electrical engineering degree from the Polytechnic University of Catalonia. Currently he is working at Sema Group Spain where he leads the satellite group.

Klaus-Jürgen Schulz obtained the Ph.D. in informatics from the University of Zurich. Currently he is working at European Space Agency (ESA) in the European Space Operations Centre (ESOC) where he participates in projects related with the communication systems at the ground segment. 